\title{
ABSOLUTE $C^{*}$-EMBEDDING OF EXTREMALLY DISCONNECTED SPACES
}

\author{
ALAN DOW
}

\begin{abstract}
An extremally disconnected space $X$ is $C^{*}$-embedded in each extremally disconnected space in which it is embedded iff $X$ is weakly Lindelö or almost compact.
\end{abstract}

1. Introduction. All spaces under discussion are Tychonoff. A space $X$ is extremally disconnected, denoted ED, if disjoint open subsets of $X$ have disjoint closures in $X$. A space is weakly Lindelöf if every open cover has a countable subcollection whose union is dense [CHN]. It is well known and easy to prove that a weakly Lindelöf subspace $X$ of an ED space $Y$ is $C^{*}$-embedded in $Y$. It is also well known that almost compact subspaces are always $C^{*}$-embedded. In this paper we prove the converse; if an ED space $X$ is not weakly Lindelöf nor almost compact then there is an ED space $Y$ such that $X$ can be embedded in $Y$ so that it is not $C^{*}$-embedded. This question was raised by C. E. Aull [A]. Our method of proof is a generalization of a technique of Balcar and Simon [BS].

2. Preliminaries. We shall need some facts regarding ED spaces and the absolute of a space.

THEOREM 2.1 (1H AND 6M OF [GJ]). The following are equivalent for a space $X$.

(1) $X$ is $E D$.

(2) $\beta X$ is $E D$.

(3) Each dense subspace of $X$ is ED.

(4) Each open subset of $X$ has open closure in $X$.

Let $Y$ be any regular space. Let $R(Y)$ be the boolean algebra of regular closed subsets of $Y$ and let $S(R(Y))$ be the Stone space of $R(Y)$. The absolute of $Y$, denoted $E(Y)$, is the subspace of $S(R(Y))$ consisting of all convergent maximal filters of $R(Y)$. The map $k: E(Y) \rightarrow Y$ which is the canonical map from $E(Y)$ to $Y$ takes each convergent maximal filter of $R(Y)$ to its limit in $Y$. This map is continuous, perfect and irreducible. The space $E(Y)$ is extremally disconnected. The reader may refer to [Wo] for more details concerning the absolute.

A base for the clopen subsets of $E(Y)$ is $\left\{\operatorname{cl}_{E(Y)} k^{\leftarrow}\right.$ [int $\left.\left.U\right]: U \in R(Y)\right\}$. It can be shown that $\mathrm{cl}_{E(Y)} k^{\leftarrow}[$ int $U$ ], for $U \in R(Y)$, is the set of all convergent maximal filters of $R(Y)$ containing $U$. We also note that $k\left[\mathrm{cl}_{E(Y)} k^{\leftarrow}[\right.$ int $\left.U]\right]=U$.

Received by the editors January 26, 1980 and, in revised form, April 14, 1980.

AMS (MOS) subject classifications (1970). Primary 54D35.

Key words and phrases. Extremally disconnected, $P$-set, the absolute. 
Let $F$ be any index set and let $2=\{0,1\}$ denote the two point discrete space. If $J$ is a finite subset of $F$ and $\psi$ is a function from $J$ into 2 , we will denote $\left\{f \in 2^{F}\right.$ : $f \supset \psi\}$ by $[\psi]$. The set $[\psi]$ is a clopen subset of $2^{F}$ and the set of all such sets is a base for the topology on $2^{F}$. Recall that $2^{F}$ is ccc.

Efimov (Remark 8, p. 274 of [E]) has shown that if $X$ is an ED space, there exists a discrete space $D$ such that $\beta X$ can be embedded into $\beta D$. In Lemma 2.3 we will show that $\beta X$ can be embedded into $\beta D$ satisfying a further property which we shall require. A subset $P$ of a space $X$ will be called a $P$-set of $X$ if $P$ is compact and the intersection of countably many neighborhoods of $P$ is again a neighborhood of $P$. An equivalent definition is that $P$ is compact and any disjoint $F_{\sigma}$ has its closure disjoint from $P$. We will use the notation " $X \bmod (P)$ " to represent the quotient space of $X$ obtained by collapsing $P$ to a point (even if $P$ is not a $P$-set).

LEMMA 2.2 (10.50 of [W]). A closed irreducible mapping of a Hausdorff space onto an extremally disconnected space is a homeomorphism.

Lemma 2.3. Let $X$ be an extremally disconnected space and let $P$ be a $P$-set of $\beta X$ that is contained in $\beta X-X$. Let $D$ be the discrete space with the same cardinality as $X$. Then there exist an embedding $h: \beta X \rightarrow \beta D$ and a compact $P$-set $P^{\prime}$ of $\beta D$ such that $P^{\prime} \dot{\cap} h[\beta X]=h[P]$.

Proof. Let $f$ be any one-to-one map of $D$ onto $X$. Then $\beta f$ is a continuous map from $\beta D$ onto $\beta X$. We can choose a compact subset $K$ of $\beta D$ such that $\left.\beta f\right|_{K}$ is a homeomorphism from $K$ onto $\beta X$ (Lemmas 2.1(2) and 2.2). We claim that $(\beta f)^{\leftarrow}[P]$ is a $P$-set of $\beta D$ and that $(\beta f)^{\leftarrow}[P] \cap K=\left(\left.\beta f\right|_{K}\right)^{\leftarrow}[P]$. Indeed, let $\left(C_{n}\right.$ : $n \in \omega)$ be clopen neighborhoods in $\beta D$ of $(\beta f)^{\leftarrow}[P]$. Then $\beta f\left[\beta D \backslash C_{n}\right] \cap P=\varnothing$ for each $n$. Since $P$ is a $P$-set of $\beta X,\left(\operatorname{cl}_{\beta X}\left[\cup_{n} \beta f\left[\beta D \backslash C_{n}\right]\right]\right) \cap P=\varnothing$. Therefore $(\beta f)^{\leftarrow}\left[\mathrm{cl}_{\beta X}\left(\cup_{n} \beta f\left[\beta D \backslash C_{n}\right]\right]\right) \cap P=\varnothing$. Therefore $(\beta f)^{\leftarrow}\left[\mathrm{cl}_{\beta X}\left(\cup_{n} \beta f\left[\beta D \backslash C_{n}\right]\right)\right]$ $\cap(\beta f)^{\leftarrow}[P]=\varnothing$. Also $\beta D \backslash C_{n}$ is contained in $(\beta f)^{\leftarrow}\left[\beta f\left[\beta D \backslash C_{n}\right]\right]$. Hence $\operatorname{cl}_{\beta D}\left(\cup_{n}\left(\beta D \backslash C_{n}\right)\right)$ is contained in $(\beta f)^{\leftarrow}\left[\operatorname{cl}_{\beta X}\left(\cup_{n} \beta f\left[\beta D \backslash C_{n}\right]\right)\right]$ and therefore $\cap_{n} C_{n}$ is a neighborhood of $(\beta f)^{\leftarrow}[P]$. Clearly $(\beta f)^{\leftarrow}[P] \cap K=\left(\left.\beta f\right|_{K}\right)^{\leftarrow}[P]$. We define $h$ to be $\left(\left.\beta f\right|_{K}\right)^{\leftarrow}$ and $P^{\prime}$ to be $(\beta f)^{\leftarrow}[P]$, and the theorem is proved.

3. The main result. The crucial step in our main result is Theorem 3.1. We will construct an embedding of a discrete space $D$ into $E\left(2^{F}\right)$, where $F$ is the power set of $D$. We will do this in such a way that the closure in $E\left(2^{F}\right)$ of our embedded copy of $D$ will be the quotient space of $\beta D$ with a specified $P$-set collapsed to a point. As noted in the introduction, our technique was developed by Balcar and Simon [BS]. In the proof of 3.1 we will use the following notation. If $D$ is a discrete space and $X$ is any space with a subset $\left\{x_{d}: d \in D\right\}$, then $x$ is a $q$-limit of $\left\{x_{d}: d \in D\right\}$, for $q \in \beta D \backslash D$ if $x \in \cap_{C \in q} \operatorname{cl}_{X}\left\{x_{d}: d \in C\right\}$.

Theorem 3.1. Let $D$ be any discrete space, let $P$ be a $P$-set of $\beta D$, and let $q$ be any element of $\beta D \backslash D$. Then $\beta D \bmod (P \cup\{q\})$ is homeomorphic to a subspace of $E\left(2^{F}\right)$ where

$$
F=\left\{C \subseteq D: \operatorname{cl}_{\beta D} C \cap(P \cup\{q\})=\varnothing\right\}
$$


Proof. First we embed $D$ in $2^{F}$. For each $d \in D$, define $x_{d} \in 2^{F}$ as follows: for $C \in F, x_{d}(C)=1$ iff $d \in C$. The set $\left\{x_{d}: d \in D\right\}$ is a faithfully indexed discrete subspace of $2^{F}$. We will assume for the remainder of the proof that $q \notin P$; one can simply ignore all mention of $q$ throughout the proof if $q \in P$.

Fact 1. If $C \subseteq D$ and $\left(\mathrm{cl}_{\beta D} C\right) \cap P=\varnothing$ then $\left\{x_{d}: d \in C\right\}$ is $C^{*}$-embedded in $2^{F}$. To prove this, let $A$ and $B$ be complementary subsets of $C$. Without loss of generality $q \notin \operatorname{cl}_{\beta D} A$. Hence $A \in F$. Therefore if we let $\tilde{A}=\left\{f \in 2^{F}: f(A)=1\right\}$ then $\tilde{A}$ is a clopen subset of $2^{F}$ such that $\tilde{A} \cap\left\{x_{d}: d \in C\right\}=\left\{x_{d}: d \in A\right\}$. Therefore $C$ is $C^{*}$-embedded in $2^{F}$, and Fact 1 is verified.

Now we choose a subset $D_{0}$ of $D$ such that $\left(\operatorname{cl}_{\beta D} D_{0}\right) \cap P=\varnothing$ and $q \in \operatorname{cl}_{\beta D} D_{0}$. By Fact $1\left\{x_{d}: d \in D_{0}\right\}$ is $C^{*}$-embedded in $2^{F}$. Recall that $k$ is the canonical map from $E\left(2^{F}\right)$ onto $2^{F}$. For each $d \in D_{0}$, choose an element $\mathscr{U}_{d}$ of $k^{\leftarrow}\left(x_{d}\right)$. The set $\left\{\mathscr{U}_{d}: d \in D_{0}\right\}$ is $C^{*}$-embedded in $E\left(2^{F}\right)$; to see this note that $k$ is a continuous map from $\mathrm{cl}_{E\left(2^{F}\right)}\left\{\mathscr{U}_{d}: d \in D_{0}\right\}$ onto $\mathrm{cl}_{2^{F}}\left\{x_{d}: d \in D_{0}\right\}$. The fact that $\left\{x_{d}: d \in D_{0}\right\}$ is discrete and $C^{*}$-embedded in $2^{F}$, together with Lemma 2.2, implies that $\left\{\mathscr{Q}_{d}: d \in D_{0}\right\}$ is $C^{*}$-embedded in $E\left(2^{F}\right)$. We can therefore find Q $\in \operatorname{cl}_{E\left(2^{F}\right)}\left\{\mathscr{U}_{d}: d \in D_{0}\right\}$ such that $\{\mathscr{U}\}=\bigcap_{A \in q} \operatorname{cl}_{E\left(2^{F}\right)}\left\{\mathscr{Q}_{d}: d \in A\right\}$. If $C \in F$ then $D \backslash C \in q$ since $\operatorname{cl}_{\beta D} C \cap(P \cup\{q\})=\varnothing$. This implies that for each $C \in F$, $\left\{f \in 2^{F}: f(C)=0\right\}$ contains $k(U)$ since $\{k(U)\}=\bigcap_{A \in q} \operatorname{cl}_{2^{F}}\left\{x_{d}: d \in A\right\}$ and $x_{d}(C)=1$ iff $d \in C$. Therefore $k(\mho)$ is the function from $F$ to 2 which is identically 0 ; denote it by $\underline{0}$. To complete the embedding of $D$ in $E\left(2^{F}\right)$, we will now choose, for each $d \in D \backslash D_{0}$, an ultrafilter $\mathcal{U}_{d} \in k^{\leftarrow}\left(x_{d}\right)$. First we need some notation. Because, as noted earlier, $2^{F}$ satisfies the countable chain condition, for each $U \in \mathcal{Q}$, there is a countable family of clopen sets $\left[\Psi_{n}\right]$ such that $\cup_{n}\left[\Psi_{n}\right]$ is a dense subset of $U$. We will let $s(U)=U\left(\operatorname{dom} \Psi_{n}\right)$. For each $n$, dom $\Psi_{n}$ is a finite subset of $F$; hence $s(U)$ is a countable subset of $F$. For each $d \in D \backslash D_{0}$ we will let $\mathscr{F}_{d}=\left\{U \in \mathscr{Q}: C \in s(U)\right.$ implies $\left.x_{d}(C)=0\right\}$.

Fact 2. If $U_{1}, U_{2}, \ldots, U_{n}$ are all in $\mathscr{F}_{d}$ for some $d \in D \backslash D_{0}$ then $x_{d} \in$ $\mathrm{cl}_{2^{F}}$ int $_{2^{F}} \bigcap_{i=1}^{n} U_{i}$.

To prove Fact 2 we first observe that, by the definition of $\mathscr{F}_{d}$, for each $C \in \cup{ }_{i=1}^{n} s\left(U_{i}\right), x_{d}(C)=0$. Let [ $\Psi$ ] be a basic neighborhood of $x_{d}$ and let $\operatorname{dom} \Psi=J_{1} \cup J_{2}$ where $J_{1} \cap \cup_{i=1}^{n} s\left(U_{i}\right)=\varnothing$ and $J_{2} \subset \cup_{i=1}^{n} s\left(U_{i}\right)$. Therefore $\left[\left.\Psi\right|_{J_{2}}\right]$ is a neighborhood of $\underline{0}$. Notice, also, that $U_{i} \in \mathcal{Q}$ for $i=1,2, \ldots, n$ and therefore $\underline{0} \in \mathrm{cl}_{2^{F}}$ int $\hat{2}^{F} \cap_{i=1}^{n} U_{i}$. Hence we can find basic clopen sets $\left[\Psi_{i}\right]$ for $i=1, \ldots, n$ with $\left[\Psi_{i}\right] \subseteq U_{i}$, dom $\Psi_{i} \subseteq s\left(U_{i}\right)$ and $\left[\left.\Psi\right|_{J_{2}}\right] \cap\left[\Psi_{1}\right] \cap \cdots \cap\left[\Psi_{n}\right] \neq$ $\varnothing$. Let $f$ be an element of $\left[\left.\Psi\right|_{J_{2}}\right] \cap\left[\Psi_{1}\right] \cap \cdots \cap\left[\Psi_{n}\right]$ and define $\hat{f}(C)=f(C)$ for $C \notin J_{1}$ and let $\hat{f}(C)=\Psi(C)$ for $C \in J_{1}$. Since $\left(\operatorname{dom} \Psi_{i}\right) \cap J_{1}=\varnothing$ for each $i$ we conclude that $\hat{f} \in[\Psi] \cap\left[\Psi_{1}\right] \cap \cdots \cap\left[\Psi_{n}\right]$. Hence $x_{d} \in \operatorname{cl}_{2^{F}}$ int $_{2^{F}} \cap_{i=1}^{n} U_{i}$ and Fact 2 is verified.

It follows from Fact 2 that $\mathscr{F}_{d}$ is a filter base in $R\left(2^{F}\right)$ and that $x_{d}$ is an accumulation point of $\mathscr{F}_{d}$. Therefore using a Zorn's lemma argument we can choose an ultrafilter $\mathscr{U}_{d}$ such that $\mathscr{U}_{d}$ contains $\mathscr{F}_{d}$ and $k\left(\mathscr{U}_{d}\right)=x_{d}$.

Fact 3. For any $U \in \mathscr{U}$ there is an $A \subseteq D$ such that $\operatorname{cl}_{\beta D} A \supset P$ and $\{\mathscr{V} \in$ $\left.E\left(2^{F}\right): U \in \mathfrak{V}\right\} \supset\left\{\mathcal{U}_{d}: d \in A\right\}$. 
To prove Fact 3, let $U$ be an element of $\mathcal{Q}$ and list $s(U)$ as $\left\{C_{n}: n \in \omega\right\}$. Each $C_{n}$ is in $F$; hence $\left(\mathrm{cl}_{\beta D} C_{n}\right) \cap P=\varnothing$. Let $A=D \backslash\left(\cup_{n} C_{n} \cup D_{0}\right)$; then $\operatorname{cl}_{\beta D} A$ contains $P$, since $P$ is a $P$-set of $\beta D$ and cl $_{\beta D} D_{0} \cap P=\varnothing$. For each $d \in A$ and $n \in \omega, x_{d}\left(C_{n}\right)=0$ which means that $U \in \mathscr{F}_{d}$. Therefore $U \in \mathcal{U}_{d}$ and $\mathcal{U}_{d} \in\{\mathcal{V}$ $\left.\in E\left(2^{F}\right): U \in \mathfrak{V}\right\}$ and Fact 3 is verified.

To complete the proof of the theorem, we will now show that $\mathrm{cl}_{E\left(2^{F}\right)}\left\{U_{d}: d \in D\right\}$ is homeomorphic to $\beta D \bmod (P \cup\{q\})$.

Fact 4. $\mathrm{cl}_{E\left(2^{F}\right)}\left\{Q_{d}: d \in D\right\}$ is homeomorphic to $\beta D \bmod (P \cup\{q\})$. First we will show that $\mathrm{cl}_{2^{F}}\left\{x_{d}: d \in D\right\}$ is homeomorphic to $\beta D \bmod (P \cup\{q\})$. Let $g$ denote the map which takes each $d \in D$ to $x_{d} \in 2^{F}$. Then $\beta g$ is a map from $\beta D$ onto $\mathrm{cl}_{2^{F}}\left\{x_{d}: d \in D\right\}$. We have already seen that $\underline{0}$ is the $q$-limit of $\left\{x_{d}: d \in D\right\}$; one can similarly show that, for any $p \in P, \underline{0}$ is the $p$-limit of $\left\{x_{d}: d \in D\right\}$ as well. Also, for any $t \in \beta D \backslash(P \cup\{q\}), \underline{0}$ is not the $t$-limit of $\left\{x_{d}: d \in D\right\}$. To see this, note that for any such $t$ there exists a $C \in t$ such that $\operatorname{cl}_{\beta D} C \cap(P \cup\{q\})=\varnothing$ and thus $\left\{f \in 2^{F}: f(C)=1\right\}$ is a neighborhood of the $t$-limit of $\left\{x_{d}: d \in D\right\}$ which does not contain $\underline{0}$. These facts together with Fact 1 imply that $\beta g$ is a map from $\beta D$ onto $\operatorname{cl}_{2^{F}}\left\{x_{d}: d \in D\right\}$ such that $\beta g[P \cup\{q\}]=\underline{0}$ and otherwise $\beta g$ is one-to-one.

Now let us consider the map $k$ restricted to $\operatorname{cl}_{E\left(2^{F}\right)}\left\{{\chi_{d}}_{d}: d \in D\right\}$. To complete the proof of Fact 4 it suffices to show that the preimage of $\underline{0}$ under $k \mid \mathrm{cl}_{E\left(2^{F}\right)}\left\{\vartheta_{d}: d \in D\right\}$ is a singleton. Recall that $\mathcal{Q}$ was chosen in $\operatorname{cl}_{E\left(2^{F}\right)}\left\{\vartheta_{d}: d \in D\right\}$ so that $k(\mathcal{Q})=\underline{0}$. Suppose that $\mathscr{V} \in \mathrm{cl}_{E\left(2^{F}\right)}\left\{\bigcup_{d}: d \in D\right\}$ and $\mathcal{V} \neq Q$. We can therefore choose $U \in \mathcal{U}$ such that $U \notin \mathcal{V}$. By Fact 3 there is an $A \subset D$ such that $\operatorname{cl}_{\beta D} A \supset P$ and $\left\{\mho \in E\left(2^{F}\right): U \in W\right\} \supset\left\{\bigcup_{d}: d \in A\right\}$. Also since $\mathcal{Q}$ is the $q$-limit of $\left\{\mathcal{U}_{d}: d \in D\right\}$ and $\left\{\mathscr{W} \in E\left(2^{F}\right): U \in \mathscr{W}\right\}$ is a clopen neighborhood of $\mathscr{Q}$ we can assume $A \in q$. Therefore $k(\mathcal{V})$ is an element of $\mathrm{cl}_{2^{F}}\left\{x_{d}: d \in D \backslash A\right\}$ and since $D \backslash A \in F$ we observe that $k(\mathfrak{V}) \neq 0$. Therefore $\beta D \bmod (P \cup\{q\})$ is embedded in $E\left(2^{F}\right)$. This verifies Fact 4 , and concludes the proof of the theorem.

We can now prove the theorem stated in the introduction which is our main result.

THEOREM 3.2. Let $X$ be an ED space which is not weakly Lindelöf nor almost compact. Then there is a $P$-set, $P$, of $\beta X$ and a point $q \in \beta X \backslash X$ such that $|P \cup\{q\}|>1$. Also $\beta X \bmod (P \cup\{q\})$ can be embedded into $E\left(2^{\lambda}\right)$ where $\lambda=2^{|X|}$.

Proof. Since $X$ is not weakly Lindelöf there is a cover $\mathcal{C}$ of $X$ consisting of clopen sets so that no countable subcollection of $\mathcal{C}$ has dense union. Define $P \subset \beta X$ to be $\cap\left\{\beta X \backslash \mathrm{cl}_{\beta X}[\cup \mathcal{E}]: \mathcal{E}\right.$ is a countable subcollection of $\left.\mathcal{C}\right\}$. One can check that $P$ is a $P$-set of $\beta X$ and that $P \subseteq \beta X \backslash X$. Since $X$ is not almost compact, $|\beta X \backslash X|>1$ [GJ, 6J]; hence a point $q \in \beta X \backslash X$ can be chosen to ensure that $|P \cup\{q\}|>1$. By Lemma 2.3 we can embed $\beta X$ into $\beta D$, with $|D|=|X|$, such that $P$ is the restriction to $\beta X$ of a $P$-set, $P^{\prime}$, of $\beta D$. We now observe that by 3.1 we can regard $\beta D$ mod $\left(P^{\prime} \cup\{q\}\right)$ as being a subspace of $E\left(2^{F}\right)$ where $|F|=2^{|D|}$. This completes the proof. 
THEOREM 3.3. For an ED space $X$, the following are equivalent:

(a) $X$ is weakly Lindelöf or almost compact,

(b) $X$ is $C^{*}$-embedded in each $E D$ space containing it,

(c) $X$ is $C^{*}$-embedded in each $E D$ space containing it as a closed subspace.

Proof. (a) implies (b) and (b) implies (c) are obvious. For (c) implies (a) let $X$ be an ED space which is not weakly Lindelöf nor almost compact. Let $P$ and $q$ be as in 3.2, then $\beta X \bmod (P \cup\{q\})$ can be embedded in the ED space $E\left(2^{\lambda}\right)$ for an appropriate $\lambda$. Clearly $Y=E\left(2^{\lambda}\right) \backslash[\operatorname{cl} X \backslash X]$ is a dense subset of $E\left(2^{\lambda}\right)$ and is therefore a $C^{*}$-embedded ED subspace of $E\left(2^{\lambda}\right)$ by 2.1. Thus $X$ is a closed subset of $Y$ but is not $C^{*}$-embedded because $X$ is not $C^{*}$-embedded in $\operatorname{cl}_{E\left(2^{\lambda}\right)} X=$ $\beta X \bmod (P \cup\{q\})$.

We remark in connection with Theorem 3.3 that an almost compact ED space of nonmeasurable cardinality is weakly Lindelöf. To see this we note that an extremally disconnected space $X$ which is not weakly Lindelöf has a $P$-set in its growth. If in addition, $X$ is almost compact, this $P$-set is a singleton, hence a $P$-point. Isbell has shown that an extremally disconnected space of nonmeasurable cardinality has no nonisolated $P$-points (12H of [GJ], [I]).

\section{REFERENCES}

[A] C. E. Aull, Absolute $C^{*}$-embedding of P-spaces, Bull. Acad. Polon. Sci. Sér. Sci. Math. 26 (1978), 831-836.

[BS] B. Balcar and P. Simon, Convergent nets in the spaces of uniform ultrafilters, (preprint) 1978.

[CHN] W. W. Comfort, N. Hindman and S. Negrepontis, $F^{\prime}$-spaces and their product with $P$-spaces, Pacific J. Math. 28 (1969), 489-502.

[E] B. A. Efimov, Extremally disconnected spaces and absolutes, Trans. Moscow Math. Soc. 23 (1970), 243-285.

[GJ] L. Gillman and M. Jerison, Rings of continuous functions, Van Nostrand, Princeton, N. J., 1960.

[I] J. R. Isbell, Zero-dimensional spaces, Tôhoku Math. J. 7 (1955), 7-8.

[W] R. C. Walker, The Stone-Cech compactification, Springer-Verlag, Berlin and New York, 1974.

[Wo] R. G. Woods, A survey of absolutes of topological spaces, Top. Structures 2, Math. Centre Tracts, no. 116,1979 , pp. $323-362$.

Department of Mathematics, University of Manttoba, Winnipeg, Manttoba, Canada R3T 2N2

Current address: Department of Mathematics, University of Kansas, Lawrence, Kansas 66045 